\title{
Transaxillary Subpectoral Placement of Cardiac Implantable Electronic Devices in Young Female Patients
}

\author{
Joo Hyun $\mathrm{Oh}^{1}$, Chae Min $\mathrm{Kim}^{1}$, Seung Yong Song ${ }^{1}$, Jae Sun $\mathrm{Uhm}^{2}$, Dae Hyun Lew ${ }^{1}$, \\ Dong Won Lee ${ }^{1}$ \\ Departments of ${ }^{1}$ Plastic and Reconstructive Surgery and ${ }^{2}$ Cardiology, Severance Hospital, Yonsei University College of Medicine, Seoul, Korea
}

Background The current indications of cardiac implantable electronic devices (CIEDs) have expanded to include young patients with serious cardiac risk factors, but CIED placement has the disadvantage of involving unsightly scarring and bulging of the chest wall. A collaborative team of cardiologists and plastic surgeons developed a technique for the subpectoral placement of CIEDs in young female patients via a transaxillary approach.

Methods From July 2012 to December 2015, subpectoral CIED placement via an axillary incision was performed in 10 young female patients, with a mean age of 25.9 years and mean body mass index of $20.1 \mathrm{~kg} / \mathrm{m}^{2}$. In the supine position, with the patient's shoulder abducted, an approximately $5-\mathrm{cm}$ linear incision was made along one of the deepest axillary creases. The submuscular plane was identified at the lateral border of the pectoralis major, and the dissection continued over the clavipectoral fascia until the subpectoral pocket could securely receive a pulse generator. Slight upward dissection also exposed an entrance to the subclavian vein, allowing the cardiology team to gain access to the vein. One patient with dilated cardiomyopathy underwent augmentation mammoplasty and CIED insertion simultaneously.

Results One case of late-onset device infection occurred. All patients were highly satisfied with the results and reported that they would recommend the procedure to others.

Conclusions With superior aesthetic outcomes compared to conventional methods, the subpectoral placement of CIEDs via a transaxillary approach is an effective, single-incision method to hide operative scarring and minimize bulging of the device, and is particularly beneficial for young female or lean patients.

Keywords Defibrillators, implantable / Pacemaker, artificial / Cardiac resynchronization therapy

Received: 22 Apr $2016 \bullet$ Revised: 5 Oct $2016 \bullet$ Accepted: 19 Oct 2016

pISSN: 2234-6163 • elSSN: 2234-6171 • https://doi.org/10.5999/aps.2017.44.1.34 • Arch Plast Surg 2017;44:34-41
Correspondence: Dong Won Lee Institute for Human Tissue Restoration, Department of Plastic and Reconstructive Surgery, Severance Hospital, Yonsei University College of Medicine, 50-1 Yonsei-ro,

Seodaemun-gu, Seoul 03722, Korea

Tel: +82-2-2228-2215

Fax: +82-2-393-6947

E-mail:XYPHOSS@yuhs.ac

This study was presented at the Sixth Research and Reconstructive Forum of the Korean Society of Plastic Surgeons on April 7th, 2016 in Daegu, Korea.

No potential conflict of interest relevant to this article was reported.

\section{INTRODUCTION}

Cardiac implantable electronic devices (CIEDs) are an essential part of cardiologic treatment in high-risk patients with a wide range of structural or functional heart diseases $[1,2]$. Over the last few decades, numerous procedural changes have been made 
in pacemaker implantation, mostly attributable to technological advancements throughout multiple generations of the devices [3]. The size of the pulse generators has also decreased over time, making it possible, most notably in pediatric patients, to replace abdominal implantation of an epicardial pacemaker with an endocardial device in the prepectoral or subpectoral space.

\section{Fig. 1. Cardiac device in a conventional subclavian pocket}

Conspicuous scarring and disfiguring bulging often result from the conventional subcutaneous insertion of cardiac implantable electronic devices in the subclavian area.

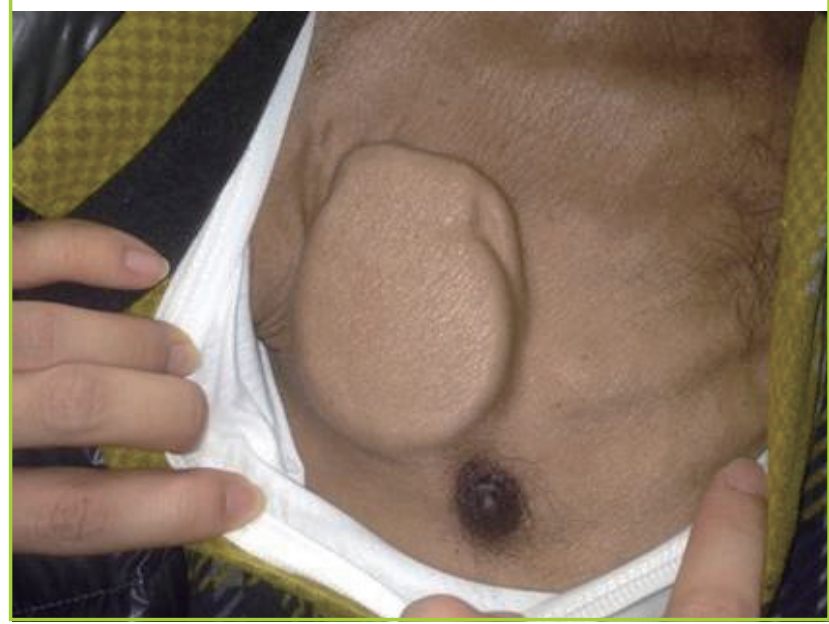

The clinical use of CIEDs has expanded to include not only middle-age patients with chronic conditions, but also younger patients predisposed to sudden cardiac death. However, several problems have arisen with conventional subcutaneous device insertion in the subclavian area, most notably the conspicuous contour of the pacemaker in the subcutaneous pocket of the anterior chest and/or an often unsightly surgical scar (Fig. 1) [4].

With this problem in mind, a collaborative team of cardiologists and plastic surgeons in our institution developed a technique for the subpectoral placement of CIEDs in young female patients via a transaxillary approach. This transaxillary approach has been frequently utilized in augmentation mammoplasty to hide visible operative scars in the axillary fold, and therefore our procedure can be deemed an extension of a familiar surgical approach for a different purpose. We present our experience with this procedure in 10 young female patients, including 1 patient who received augmentation mammoplasty together with CIED placement.

\section{METHODS}

\section{Patients}

From July 2012 to December 2015, subpectoral CIED placement via the transaxillary approach was performed in 10 young

\section{Table 1. Patient demographics, cardiologic indications for CIED implantation with device information, and postoperative follow-up periods}

\begin{tabular}{|c|c|c|c|c|c|c|c|c|c|}
\hline $\begin{array}{l}\text { Patient } \\
\text { no. }\end{array}$ & $\begin{array}{l}\text { Age } \\
(y r)\end{array}$ & $\begin{array}{l}\text { Height } \\
\text { (cm) }\end{array}$ & $\begin{array}{l}\text { Weight } \\
\text { (kg) }\end{array}$ & $\begin{array}{c}\text { BMI } \\
\left(\mathrm{kg} / \mathrm{m}^{2}\right)\end{array}$ & Symptom (s) & Indication & Device & $\begin{array}{l}\text { Model, company, and } \\
\text { country }\end{array}$ & $\begin{array}{l}\text { Follow-up } \\
\text { (wk) }\end{array}$ \\
\hline 1 & 38 & 151.6 & 53.5 & 23.28 & Palpitation, chest discomfort & Sick sinus syndrome & Pacemaker & Evia, Biotronik, Germany & 214.4 \\
\hline 2 & 29 & 166 & 55 & 19.96 & Sudden cardiac arrest & Ventricular fibrillation & ICD & $\begin{array}{l}\text { Incepta ICD, Boston Scientific, } \\
\text { United States }\end{array}$ & 130.4 \\
\hline 3 & 34 & 155 & 51 & 21.09 & Syncope & Dilated cardiomyopathy & ICD & $\begin{array}{l}\text { Ellipse DR, St. Jude Medical, } \\
\text { United States }\end{array}$ & 126.0 \\
\hline 4 & 16 & 162 & 49 & 18.67 & Sudden cardiac arrest & Dilated cardiomyopathy & ICD & $\begin{array}{l}\text { Ellipse DR, St. Jude Medical, } \\
\text { United States }\end{array}$ & 76.0 \\
\hline 5 & 22 & 151 & 51.9 & 22.49 & Dizziness & $\begin{array}{l}\text { Chemotherapy-induced } \\
\text { dilated cardiomyopathy }\end{array}$ & CRT-D & $\begin{array}{l}\text { VivaQuad XT CRT-D, Medtronic, } \\
\text { Ireland }\end{array}$ & 65.4 \\
\hline 6 & 37 & 154 & 48 & 20.24 & Syncope & $\begin{array}{l}\text { High-degree atrioventricular } \\
\text { block }\end{array}$ & Pacemaker & $\begin{array}{l}\text { Accolade EL, Boston Scientific, } \\
\text { United States }\end{array}$ & 59.1 \\
\hline 7 & 18 & 160 & 48.3 & 18.75 & Syncope & Sick sinus syndrome & Pacemaker & $\begin{array}{l}\text { Accolade EL, Boston Scientific, } \\
\text { United States }\end{array}$ & 57.4 \\
\hline 8 & 25 & 153 & 43 & 18.37 & Syncope & Hypertrophic cardiomyopathy & ICD & $\begin{array}{l}\text { Evera MRI XT DR, Medtronic, } \\
\text { Ireland }\end{array}$ & 32.4 \\
\hline 9 & 20 & 162.1 & 53 & 20.17 & Dyspnea, dizziness & $\begin{array}{l}\text { High-degree atrioventricular } \\
\text { block }\end{array}$ & Pacemaker & $\begin{array}{l}\text { Advisa DR MRI, Medtronic, } \\
\text { Ireland }\end{array}$ & 36.3 \\
\hline $10^{\text {a) }}$ & 20 & 161 & 45.2 & 17.44 & Dyspnea & Dilated cardiomyopathy & ICD & $\begin{array}{l}\text { Ellipse DR, St. Jude Medical, } \\
\text { United States }\end{array}$ & 84.1 \\
\hline Mean & 25.9 & 157.6 & 49.8 & 20.05 & - & - & - & & 88.2 \\
\hline
\end{tabular}

CIED, cardiac implantable electronic device; BMI, body mass index; ICD, implantable cardioverter-defibrillator; CRT-D, cardiac resynchronization therapy defibrillator. ${ }^{a)} \mathrm{A}$ patient who received concomitant augmentation mammoplasty together with CIED placement. 
female patients who were referred from a group of 3 cardiologists at our institution (Table 1).

All subjects were young and lean female patients with a mean age of 25.9 years (range, 16-38 years) and a mean body mass index (BMI) of $20.1 \mathrm{~kg} / \mathrm{m}^{2}$ (range, $17.44-23.28 \mathrm{~kg} / \mathrm{m}^{2}$ ). The mean postoperative follow-up period was 88.2 weeks.

The patients had been diagnosed with a range of anatomical and functional cardiac abnormalities. Five patients had been diagnosed with various types of cardiomyopathy, of whom 3 had dilated cardiomyopathy, 1 had chemotherapy-induced cardiomyopathy, and 1 patient had hypertrophic cardiomyopathy. Three patients had symptomatic sick sinus syndrome. Two patients had high-degree atrioventricular block, and 1 patient had ventricular fibrillation.

All patients were highly concerned with any noticeable physical changes on their chest following the CIED insertion, and they wanted any surgical scar to be concealed as much as possible. One patient with dilated cardiomyopathy inquired about the possibility of combining augmentation mammoplasty using the conventional endoscopic approach and CIED insertion under the same subpectoral plane, and we carried out a combination of these 2 procedures in that patient.

Each patient was informed that the inserted device would require a battery change every 10 years at the time of battery depletion, and that the same subpectoral plane would be used again during each of these future procedures.

We also reviewed all surgical complications that could lead to device removal, such as postoperative pain, device migration, lead dislodgement, and infection.

\section{Surgical technique}

All patients were under general anesthesia throughout the entire surgical procedure. In the supine position, the patient's shoulder was abducted to expose the axillary area and the lateral border of the pectoralis major muscle. On the axillary fossa, a linear incision approximately 5-7 cm in length was made along one of the deepest axillary creases, mostly perpendicular to the lateral border of the pectoralis major. Care was taken not to extend this incision beyond the anterior axillary fold. The subcutaneous dissection proceeded, with undermining remaining close to the lateral border of the pectoralis major muscle, since major neurovascular structures are located posteriorly under the axillary fat pad. The scrub assistants, if any, should be informed not to retract the lateral tissue too strongly, because this can stretch the intercostobrachial nerve and the patient may complain of numbness of the upper medial aspect of the affected arm after surgery.

When the fascia of the pectoralis major was encountered at its
Fig. 2. Device positioning using the transaxillary approach

The device is placed inside the subpectoral pocket along the midclavicular level, and is fixated with absorbable sutures onto the chest wall. A slight upward dissection provides a window to the subclavian vein through which the atrial and ventricular leads are introduced.

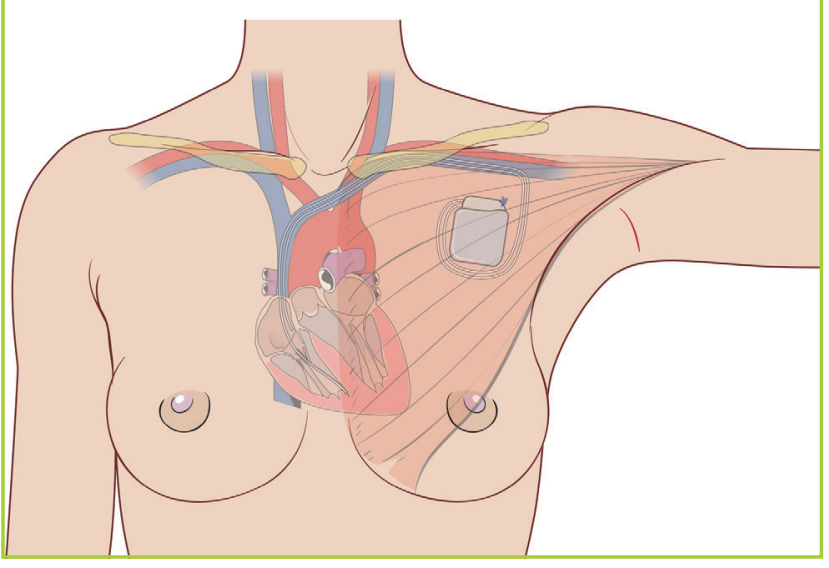

lateral border, the submuscular plane was identified while maintaining intact attachment of the pectoralis minor muscle to the chest wall. The clavipectoral fascia enclosing the pectoralis minor muscle can be readily differentiated from the pectoral fascia of the pectoralis major. The dissection continued over the clavipectoral fascia; otherwise, further dissection under the pectoralis minor may damage vital structures, such as the thoracoacromial artery, cephalic vein, or lateral pectoral nerve, which may appear beyond the muscle. The medial pectoral nerve mainly supplies the pectoralis minor and partly the pectoralis major, and it may also be exposed and inadvertently damaged during undermining. This alone, however, would not result in significant functional sequelae. Under the submuscular plane of the pectoralis major, dissection is relatively easy without being disturbed by arterial perforators, and the surgeon continued until a pocket became large enough to securely receive a cardiac pulse generator. Slight upward dissection also exposed an entrance window to the subclavian vein, allowing the cardiology team to easily gain access to the vein (Fig. 2).

Using the Seldinger technique, the subclavian vein was punctured, and a cardiologist introduced the ventricular lead and, if necessary, an atrial lead via a 9-Fr guiding sheath while stably anchoring them on the right ventricular apex and right atrial appendage, respectively. The ventricular and atrial leads were then connected to a pulse generator, and device function was evaluated using wireless telemetry with the device in place. The device was then stably fixed onto either the periosteum of the rib bone or the outermost fascia of the intercostalis muscle with absorbable sutures, and 1 or 2 other additional sutures were made into the surrounding tissues (Fig. 3). A negative-pressure drain 


\section{Fig. 3. Pulse generator in the subpectoral pocket}

Intraoperative view of an implantable cardioverter-defibrillator pulse generator inside the subpectoral pocket placed via the transaxillary approach.

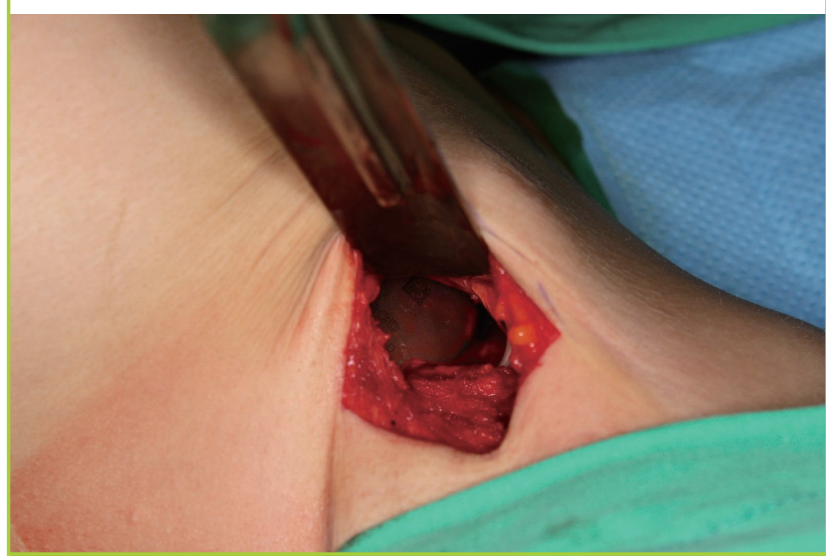

was inserted, and the subcutaneous tissue was repaired in layers, first with absorbable sutures and then with nonabsorbable sutures for skin closure (Fig. 4).

Blood pressure and other signs of hemodynamics should be carefully monitored during the entire procedure, particularly while the patient is sitting up for an evaluation of the shape and position of the implants.

Postoperative pain was usually not remarkable, and analgesia use was similar to or less than that observed in patients who undergo augmentation mammoplasty. We maintained negativepressure drains until the daily amount of drainage became less than $20 \mathrm{~mL}$.

\section{RESULTS}

\section{Cardiacimplantable electronic devices}

All patients were treated with subpectoral CIED insertion at a single institution by 2 plastic surgeons in collaboration with 3 cardiologists (Table 1). Four patients received pacemakers, 5 received implantable cardioverter defibrillators (ICDs), and 1 patient was treated with a cardiac resynchronization therapy defibrillator.

\section{Patient satisfaction}

In general, all patients were highly satisfied with the outcomes. They responded with an average of 8.5 points on a scale of 1 to 10 when asked about how much they were pleased with the benefits of the transaxillary procedure. Some patients complained of minor discomfort, such as temporary hypoesthesia on and around the incision area. Some did not like the fact that they were required to limit their arm movements for the first
Fig. 4. Immediate postoperative view of the axillary area

Immediate postoperative view after negative-pressure drainage insertion and wound closure. Note that the axillary incision did not extend beyond the anterior axillary fold.

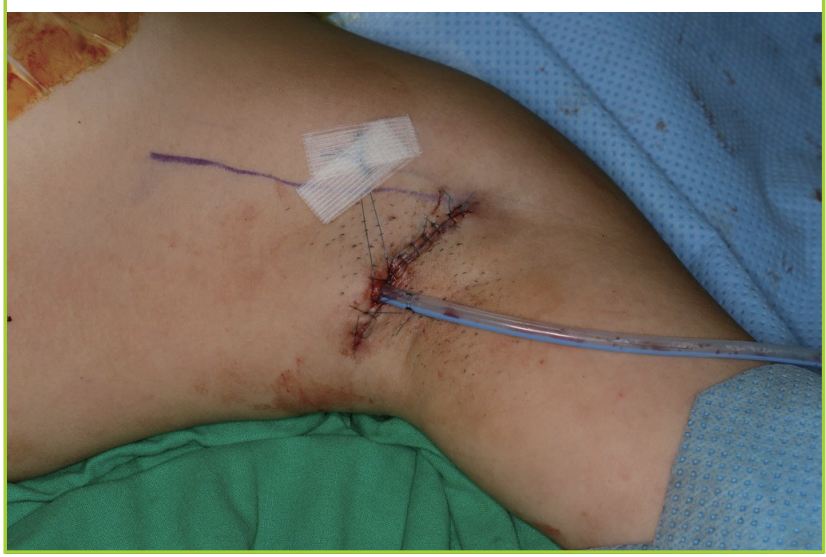

few postoperative weeks. However, all patients said that they would recommend this procedure to others of the same age with similar cardiac conditions.

\section{Complications}

One case of device pocket infection occurred. A bacterial swab culture and subsequent blood culture revealed methicillin-resistant Staphylococcus aureus. The device was ultimately surgically removed. One month after device removal, when the inflammation had subsided completely, a new device was inserted into the right anterior chest wall using the conventional subcutaneous plane.

Another patient experienced mild tingling on the medial aspect of the right upper arm, which resolved spontaneously within several weeks. No other significant complications were found in the other patients.

\section{DISCUSSION}

As implantable cardioverter defibrillators have become a mainstay of antiarrhythmic treatments for the primary and secondary prevention of sudden cardiac death, providing substantial benefits in terms of utility, efficacy, and safety $[1,2,5]$, several researchers have investigated the quality of life among recipients of these devices $[6,7]$. Moreover, the indications for CIEDs are expanding to include younger patients, with the early recognition of inherited cardiac syndromes, such as long QT syndrome, Brugada syndrome, and hypertrophic cardiomyopathy.

Once implanted, CIEDs should be maintained for the rest of the patient's life, and the physical and psychological impact can therefore be much greater among young patients. In addition, 
these patients will experience psychological discomfort if visible scars and bulging of the device are not addressed properly, leading to a more intense need to alleviate the stigma experienced by patients.

The psychosocial impact of CIEDs can be of particular concern, making young female patients most vulnerable. Vazquez reported that younger female patients showed worse perceptions of shock anxiety, death anxiety, and body image distress than older female patients [4]. In 2012, Marshall et al. [8] reported that female patients were more distressed than male patients about the impact of CIEDs on their appearance, especially women younger than 39 years of age. Earlier research also revealed that younger CIED patients had concerns about various issues, such as the fit of clothing, socialization, and sexual activity.

The conventional technique, which involves a subclavian skin incision with subcutaneous pocket formation, fails to address these quality of life issues. CIED recipients have often been stigmatized due to the operative scar in the infraclavicular area, which is highly susceptible to hypertrophic scarring, because unusual tension is applied due to the convexity of the chest wall and weight of the breast. The superficial placement of the device also involves a greater risk of skin ulceration or device exposure.

Several alternative surgical approaches, therefore, have been suggested in response to these problems. A surgical approach through the inframammary fold was the first alternative route described in the literature in an effort to place the pacemaker or CIED behind the breast tissue or pectoralis major muscle. In 1983, Belott and Bucko [9] first presented his experience with 2 female patients utilizing an inframammary approach for pacemaker placement. Allan [10], in 1985, published a case report describing his use of the familiar inframammary approach employed in augmentation mammoplasty to implant a pacemaker. In 1993, Kolettis et al.[11] reported successful retromammary CIED device placement with an endocardial lead system in a 62-year-old woman. These early attempts were done exclusively using a subglandular pocket, followed by Ozin et al. [12] and Schaverien et al. [13].

Giudici [14] and Collegues [15], in 2001 and 2010, published his comprehensive experience with the subpectoral placement of pacemakers and CIEDs in 51 female patients over a 9-year period. He used the subpectoral plane to place the devices with the skin incision on the inframammary fold for device insertion and a separate 15-20-mm incision on the anterior axillary line for lead insertion. Patient satisfaction was high, and 3 cases of lead dislodgement and 1 case of pneumothorax were reported as complications. Persichetti also presented, in 2014, 30 consecutive female patients who underwent submuscular implantation or substitution of CIED devices, which was the largest clinical series for submuscular ICD implantation [16]. The inframammary approach leads to excellent cosmetic results, since the operative scar is seen only when the patient raises her arm. However, as a 2-incision method, it still requires another skin incision in the infraclavicular area for lead insertion and narrow subcutaneous tunneling to the device pocket behind the breast.

Other alternative routes include subpectoral placement via a conventional infraclavicular incision. In 1992, Hammel et al. [17] inserted a CIED under the pectoralis major with a cephalic venotomy incision in an obese woman. In 2014, Asamura et al. [18] presented results from 21 male patients for the secondary replacement of CIED devices; by using the previous operative scar in the infraclavicular area, he inserted the devices under the pectoralis major muscle by splitting the muscle fibers between the clavicular and sternocostal attachment points. Half of the patients were lean, with a BMI under $18.5 \mathrm{~kg} / \mathrm{m}^{2}$, and the bulge from the device became much smoother after the procedure than is the case for conventional subcutaneous pockets, although an infraclavicular scar was still obvious.

A skin incision via the lateral border of the pectoralis muscle was also utilized by several researchers, particularly among pediatric patients. Molina [19], in 1991, published his long-term experience with 83 adult female patients and 21 pediatric patients. Two skin incisions, a short subclavian puncture and a longer lateral breast incision, were used to insert the device in the subglandular pocket in female patients. Several other reports of subpectoral pacemaker insertion for pediatric patients were published by Shefer et al. [20] in 1996 and Rosenthal [21] in 2000, using a direct skin incision along the lateral border of the pectoralis major. However, this operative scar on anterior axillary fold was still readily visible upon even slight arm abduction.

Despite the variety of these previous approaches, we believe that the transaxillary approach is the most suitable method for young female patients. It can effectively conceal the operative scar in the axillary fossa, as it can be disguised between natural axillary creases, as long as the operator does not extend the incision anteriorly beyond the anterior axillary fold. The bulge from the device is hardly noticeable, allowing the patients to continue their normal lives without continuously paying attention to the existence of the cardiac device (Fig. 5). This approach may eliminate even the small inconveniences in daily life caused by the bulge of the device when wearing a seatbelt or carrying a bag with shoulder straps.

The young female patients in our study also showed a high level of satisfaction regarding the benefits and outcomes of the procedure, and unanimously said that they would recommend this transaxillary subpectoral approach to those in similar circumstances. 


\section{Fig. 5. Postoperative results of the transaxillary technique}

$(A, B)$ Eight-month postoperative views of a lean 25-year-old female patient (body mass index $18.37 \mathrm{~kg} / \mathrm{m}^{2}$ ) with hypertrophic cardiomyopathy who underwent subpectoral placement of a cardiac implantable electronic device. A slight bulge of the device can be palpated on her left anterior chest wall, but almost unrecognizable by others. $(C, D)$ Flat and linear operative scars can be observed only if the arm is raised well over $90^{\circ}$. $\left(E_{1}\right.$ F) Two-month postoperative chest $\mathrm{X}$-ray images showing no evidence of device migration.
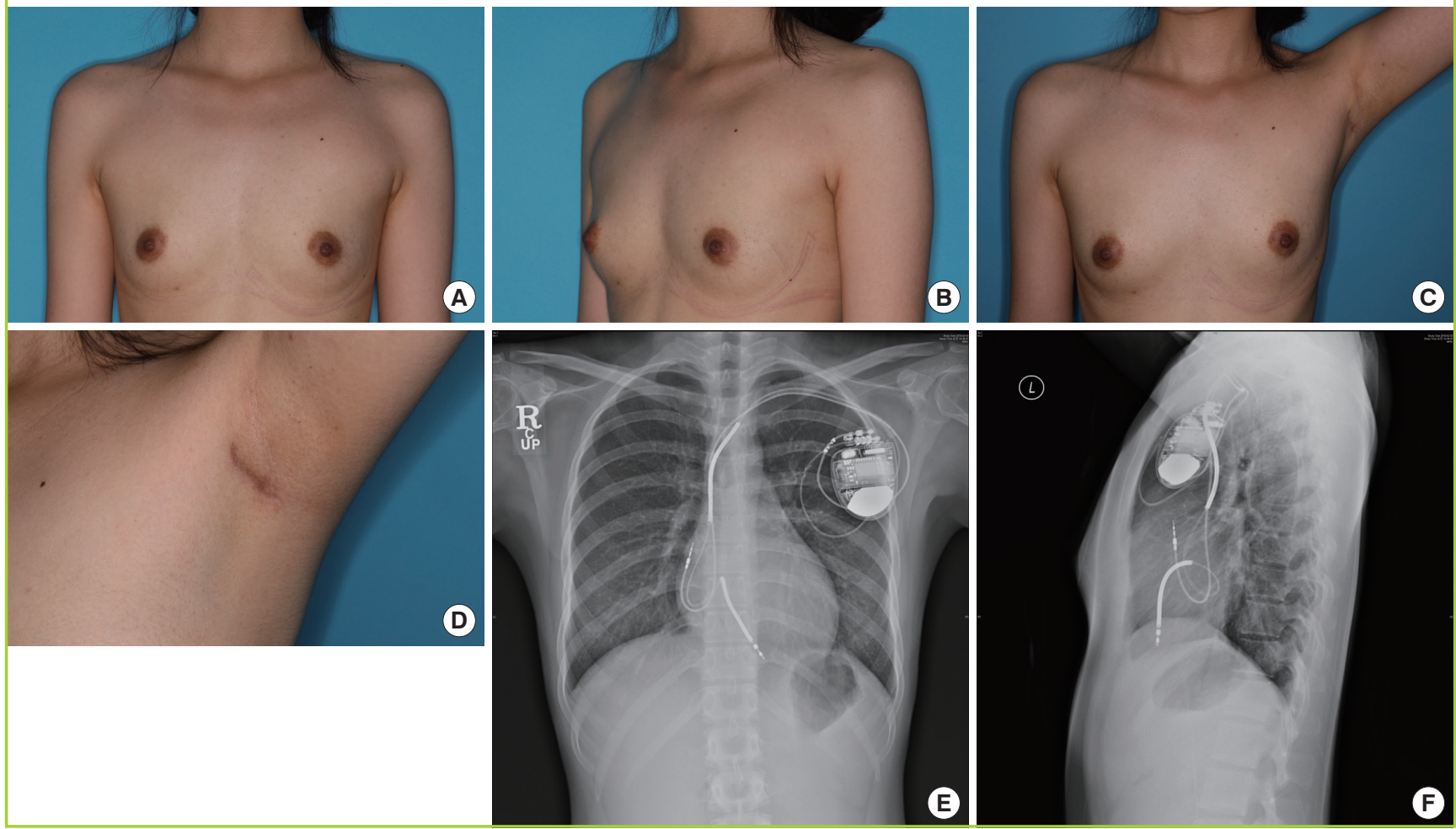

This approach is already time-tested in cosmetic plastic surgery with regard to operative scar management. Moreover, it does not necessitate a separate skin incision in the infraclavicular area, since the subclavian vein can also be approached via a single axillary incision. An additional skin stab incision, no matter how short it may be, can be a psychological burden for the patient if it is located in a potentially visible area.

The transaxillary approach is not regarded as a technically demanding task for a plastic surgeon. When performing cosmetic augmentation mammoplasty, bleeding from perforators can be troublesome given the limited surgical field, but the subpectoral pocket for CIED placement is laterally positioned and small, such that medial perforators are rarely encountered. Plastic surgeons should not find it difficult to implement this transaxillary CIED placement procedure, since it is a simpler form of an already familiar approach, only for a different purpose.

Our first patient experienced bacterial infection inside the device pocket, and the CIED eventually had to be removed. However, we encountered no other complications, such as device dislocation, lead dislodgement, or pneumothorax. Device dislocation is a very unlikely occurrence, since the device is fixated with absorbable sutures tightly onto the chest wall fascia or peri- osteum through a separate hole at the corner of the pulse generator device. Shoulder movement is also restricted for the first 2 postoperative weeks, leaving the device unaffected by pectoralis major muscle contraction, which may potentially dislocate the device to an unfavorable position.

The device must be periodically replaced upon battery depletion multiple times throughout the patients' lives. As the device in the subpectoral space sits comfortably inside a fibrous capsule by the time it is about to run out of battery, theoretically after 10 years, battery exchange can be readily performed by opening the encapsulated pocket and removing the previous device after disconnecting it from the previous leads. The leads can be used permanently, so only the pulse generator device should be exchanged and functionally tested before reinstallation. The device pocket is located at approximately the mid-clavicular level, so the dissection does not have to be deeper or more extensive than the initial procedure.

A transaxillary approach is most appropriate when a lean female patient is considering augmentation mammoplasty. One patient in this study requested a concurrent procedure combining both CIED placement and augmentation mammoplasty, and showed a successful result, with minimal bulging of the de- 
vice and no additional cardiologic risk. When the procedure is combined with augmentation mammoplasty, the surgical approach is largely identical, as the mammary implants are inserted under the same submuscular plane, but the pocket of the breast implant can be distinguished from that of the more superiorly positioned CIED. The pocket dissection should not be too extensive compared with the size and shape of the cardiac device and breast implant. Otherwise, the breast implant becomes susceptible to gradual migration.

Despite the benefits of the transaxillary approach for subpectoral placement, not all medical centers may be able to perform this procedure, since it requires a certain degree of dedication from cardiologists and plastic surgeons alike. For cardiologists, the use of a transaxillary approach in the subpectoral plane utilizes a completely different set of procedures, so they should not be expected to learn this approach in a center without plastic surgeons. This technique can be made possible by the collaborative efforts of dedicated cardiologists and plastic surgeons.

To date, this is the largest clinical series to be published on the subpectoral placement of CIEDs via a transaxillary approach, which results in superior aesthetic outcomes compared to conventional methods, because this approach is an effective, singleincision method to obscure operative scars and minimize bulging of the device. Patients do not have to be aware of the device at all times, and they can remain as socially active as before the procedure. Young female or lean patients are specific subgroups that would benefit the most from this approach.

\section{REFERENCES}

1. Connolly SJ, Hallstrom AP, Cappato R, et al. Meta-analysis of the implantable cardioverter defibrillator secondary prevention trials: AVID, CASH and CIDS studies. Antiarrhythmics vs Implantable Defibrillator study. Cardiac Arrest Study Hamburg. Canadian Implantable Defibrillator Study. Eur Heart J 2000;21:2071-8.

2. Goldberger Z, Lampert R. Implantable cardioverter-defibrillators: expanding indications and technologies. JAMA 2006;295:809-18.

3. Hammill SC, Kremers MS, Stevenson LW, et al. Review of the registry's fourth year, incorporating lead data and pediatric ICD procedures, and use as a national performance measure. Heart Rhythm 2010;7:1340-5.

4. Vazquez LD, Kuhl EA, Shea JB, et al. Age-specific differences in women with implantable cardioverter defibrillators: an international multi center study. Pacing Clin Electrophysiol 2008;31:1528-34.

5. A comparison of antiarrhythmic-drug therapy with implant- able defibrillators in patients resuscitated from near-fatal ventricular arrhythmias: the Antiarrhythmics versus Implantable Defibrillators (AVID) Investigators. N Engl J Med 1997;337:1576-83.

6. Sears SF Jr, Todaro JF, Lewis TS, et al. Examining the psychosocial impact of implantable cardioverter defibrillators: a literature review. Clin Cardiol 1999;22:481-9.

7. Thomas SA, Friedmann E, Kao CW, et al. Quality of life and psychological status of patients with implantable cardioverter defibrillators. Am J Crit Care 2006; 15:389-98.

8. Marshall P, Ketchell A, Maclean J. Comparison of male and female psychological outcomes related to implantable cardioverter defibrillators (COMFORTID). Eur J Cardiovasc Nurs 2012;11:313-21.

9. Belott PH, Bucko D. Inframammary pulse generator placement for maximizing cosmetic effect. Pacing Clin Electrophysiol 1983;6:1241-4.

10. Allan D. Augmentation mammaplasty approach to pacemaker implantation. Ann Plast Surg 1985;15:242-3.

11. Kolettis TM, Saxena A, Krol RB, et al. Submammary implantation of a cardioverter-defibrillator with nonthoracotomy lead system. Am Heart J 1993;126:1222-3.

12. Ozin B, Borman H, Bozbas H, et al. Implantation of submammary implantable cardioverter defibrillators. Pacing Clin Electrophysiol 2004;27:779-82.

13. Schaverien MV, Elder D, Munnoch DA. Inframammary cardiac pacemaker insertion in women: an aesthetic alternative. Plast Reconstr Surg 2013;131:464e-465e.

14. Giudici MC. Experience with a cosmetic approach to device implantation. Pacing Clin Electrophysiol 2001;24:1679-80.

15. Giudici MC, Carlson JI, Krupa RK, et al. Submammary pacemakers and ICDs in women: long-term follow-up and patient satisfaction. Pacing Clin Electrophysiol 2010;33: 1373-5.

16. Persichetti P, Brunetti B, Cagli B, et al. Aesthetic subpectoral placement of implantable cardioverter defibrillators. Ann Plast Surg 2014;72:188-92.

17. Hammel D, Block M, Borggrefe M, et al. Implantation of a cardioverter/defibrillator in the subpectoral region combined with a nonthoracotomy lead system. Pacing Clin Electrophysiol 1992;15:367-8.

18. Asamura S, Kurita T, Motoki K, et al. Efficacy and feasibility of the submuscular implantation technique for an implantable cardiac electrical device. Eplasty 2014;14:e40.

19. Molina JE. New technique for pacemaker implantation in the upper chest of children and women. Ann Thorac Surg 1991;51:992-5.

20. Shefer A, Lewis BS, Gang ES. The retropectoral transaxil- 
lary permanent pacemaker: description of a technique for percutaneous implantation of an "invisible" device. Pacing Clin Electrophysiol 1996;19:1646-51.
21. Rosenthal E. A cosmetic approach for pectoral pacemaker implantation in young girls. Pacing Clin Electrophysiol 2000;23:1397-400. 